\title{
The Level of Metabolic and Immunological Status of Newborn Calves Under the Action of Timogen on the Body of Down-calving Cows
}

\author{
Valerian I. Velikanov ${ }^{1}$, Andrey V. Klyapnev ${ }^{1}$, Leonid V. Kharitonov ${ }^{2}$ \\ and Alexander S. Zenkin ${ }^{3}$ and Nataliya Yu. Kalyazina ${ }^{3}$ \\ ${ }^{1}$ Nizhny Novgorod State Agricultural Academy, 97 Gagarin Prospect, \\ Nizhny Novgorod, 603107, Russian Federation \\ ${ }^{2}$ All-Russia Institute of Animal Physiology, Biochemistry and Nutrition, \\ Institute township, Borovsk 249013, Kaluga Region, Russian Federation. \\ ${ }^{3}$ Ogarev Mordovia State University, 68 Bolshevistskaya str., \\ Saransk, 430005, Republic of Mordovia, Russian Federation.
}

http://dx.doi.org/10.13005/bbra/2159

(Received: 02 April 2016; accepted: 20 May 2016)

\begin{abstract}
The purpose of the current research was to determine possible effects of timogen on accumulation of immunoglobulins and other immunogenic factors in the mammary gland of cows before calving, their release with colostrum, as well as the impact of these factors on the level of metabolic and immunological status of newborn calves after feeding them with colostrum. The data are obtained in the experiments conducted at "Arsenyevskoye" (Kaluga region) and "Mir" (Dalnekonstantinovsky district of Nizhny Novgorod region) farming enterprises. The experiment is done in the spring and summer periods of 2015 testing down-calving cows and their new-born calves. The experiment was conducted in 2 stages. The studying objects of the $1^{\text {st }}$ stage were 8 down-calving cows of black-motley breed selected based on the principle of analogs, which were divided into control and experimental groups. Cows from the experimental group were exposed to intramuscular injection of timogen at a dose of $1.2 \mathrm{mg} \mathrm{3-6}$ days before calving. Control animals were injected subcutaneously with sodium chloride physiological solution according to the same pattern. Blood for research was taken from newborn calves one day and ten days after birth. Twenty-four hours after the start of feeding newborn calves with colostrum, elevated levels of immunoglobulins, total protein and hemoglobin were observed in their blood as compared to the calves from the control group. These differences persisted during 10 days after birth. The second stage of the experiment was conducted in the farming enterprise "Mir" of the Dalnekonstantinovsky district of Nizhny Novgorod region. The studies were performed according to the same scheme. Twelve down-calving cows were selected on the basis of the principle of analogs. Besides, blood analysis was conducted using additional morphological and immunological methods. Twenty-four hours after birth and feeding with colostrum, calves born from experimental cows, showed significantly increased number of erythrocytes and leukocytes, mainly at the cost of lymphocytes, as well as a higher level of total protein and its fractions, especially $\beta$ - and $\gamma$-globulins, and increased bactericidal and lysozyme activity of blood serum.
\end{abstract}

Key words: Netabolic and immunological status, down-calving cows, newborn calves, colostrum, timogen.

One of the possible methods to increase the productivity of young cattle is enhancing the level of metabolic and immunological status and

\footnotetext{
* To whom all correspondence should be addressed.
}

resistance of the body, especially under conditions of unbalanced feeding of nursing cows and violations of the animal management technology. The main factors of the body's protection against pathogens are antibodies, which in most animals occur in amount of about $1 \%$ of blood weight ${ }^{1,2}$. Various pathological processes in the body may 
lead to decreased production of T- and $\beta$ lymphocytes, development of a more pronounced leukocyte migration inhibition test (LMIT), decrease the absorption capacity of neutrophils (by phagocytic number and phagocytic index), suppress T-helper and T-killer cells production, as well as the immune response mediators. This leads to an imbalance in the immune system, and veterinary professionals are faced with a new animal pathology - an immune deficiency and a decrease in the level of metabolic processes in the body. In such situations it is absolutely necessary to use immune response-modulating agents, correcting metabolic status and immune processes in the body. These agents increase the resistance of animals against adverse factors, enhance the immune response at the vaccination, activate the body defenses, and help to enhance the therapeutic efficacy in the application of antimicrobial, antiviral and antiparasitic drugs, stimulate the regeneration processes and possess growth promoting properties, have adaptogenic effects and correct the effect of stress factors on the organism ${ }^{3-5}$.

Many drugs are offered for these purposes; however, natural substances involved in the natural regulation of body activity have the preference ${ }^{2}$.

Previously conducted studies revealed the involvement of a number of amino acids in the regulation of digestive processes, intermediate metabolism and other metabolic processes, as well as nonspecific resistance of calves. This allowed selecting application methods of amino acids preparations for these purposes. In newborn calves, the amino acids of glycine, glutamate, taurine, and ornithine, administered orally or parenterally, increase the absorption intensity of the colostrum immunoglobulins in the intestine, and accelerate the formation of natural resistance ${ }^{3 \text {, }}$ 4-7.

Derivatives of amino acids, including peptides are also involved in the regulation of metabolic processes and immune status ${ }^{8-11}$.

Thus, correction of metabolic and immune processes in the body is relevant, complex and extensive problem in humane medicine, requiring scientifically-based practical solutions. The authors sought to determine the possible impact of timogen on the ability of accumulation of immunoglobulins and other immunogenic factors in the mammary gland of cows before calving, as well as their release with colostrum that should increase the level of metabolic and immunological status of newborn calves after feeding them with colostrum.

\section{Materials and research methods}

For research we have chosen timogen - a synthetic peptide of thymus - glutamyltryptophan. Glutamic acid, which is part of it, belongs to the group of amino acids, able to accelerate the differentiation of precursors of $\mathrm{T}$ cells into T-lymphocytes and to amplify the response to heterologous erythrocytes in vitro on the splenocytes that was revealed in the experiments with laboratory animals. Timogen stimulated humoral immune response in thymusdeprived mice, increased the contents of cyclic nucleotides in splenocytes, as well as the chemotaxis and phagocytosis of neutrophils, stimulated liver regeneration in rats after partial hepatectomy and in experimental cirrhosis ${ }^{12-15}$.

It is known that the timogen increases the immunological reactivity of the organism, strengthens the lymphoid cells differentiation processes, normalizes the number of T-helpers, Tsuppressors and their ratio in blood and lymphoid organs, as well as stimulates cellular metabolism and increases the animal growth rate ${ }^{12-14}$. The drug is prescribed in cases of immunodeficiencies, disorders of regenerative processes, for the prevention of viral and bacterial infections, to increase the immune response and animal growth rate $^{10,15}$.

Previously, we studied the effect of timogen on the absorption of colostral immunoglobulins in newborn calves. It was found that the double intramuscular injection of the timogen preparation to down-calving cows at a dose of $100 \mu \mathrm{g}$ at the $1^{\text {st }}$ and $5-6^{\text {th }}$ hours after the birth increases the level of immunoglobulins in the blood of newborn experimental calves as compared to the control animals. These differences persisted for 10 days after the birth of calves, although to a lesser extent ${ }^{6,8,11}$. To ensure a high level of metabolic and immune status in newborn calves, present studies intent to apply timogen to the pregnant cows in the last days before calving. It is known that immunoglobulins are being accumulated in the colostrum 3-9 days before calving. It is assumed that the timogen may contribute to this 
accumulation and thus provide the newborn calf with immunoglobulins and enhance the metabolic processes in his organism. This does not prevent the flow through the placenta of a number of substances that regulate protective factors of the fetus, as well as intake of these regulators with colostrum.

The experiments were performed on down-calving cows and their new-born calves at "Arsenyevskoye" (Kaluga region) and "Mir" (Dalnekonstantinovsky district of Nizhny Novgorod region) farming enterprises. The experimental work was conducted in the spring and summer of 2015 and consisted of 2 stages.

The first stage of the experiment was conducted at the "Arsenyevskoye" farming enterprise in Kaluga region. The studying objects were 8 down-calving cows of black-motley breed selected based on the principle of analogs. Selected cows were divided into control and experimental groups. Cows of the experimental group were exposed to intramuscular injection of timogen given as a single dose of 1.2 mg 3-6 days before calving. The animals from the control group were exposed to subcutaneous injection of sodium chloride physiological solution according to the same pattern. Blood for research was taken from newborn calves one day and ten days after birth. The second stage of the experiment was conducted at the "Mir" farming enterprise of Dalnekonstantinovsky district of Nizhny Novgorod region. The study was conducted according to the same scheme, though a larger number of animals (12 down-calving cows) were tested as well as additional morphological and immunological methods were applied for blood analysis.

The following indicators were determined in calves during carrying out of scientific and economic experiment:

a) hematologic indicators included in vitro counting the number of erythrocytes and leukocytes in the Goryaev chamber, determining the amount of hemoglobin by Sahli method, the excretion of leukogram by counting in blood smears of different types of white blood cells stained by Romanovsky-Gimza;

b) biochemical indicators included studying the level of total protein, blood urea and glucose in the serum of animals by means of Olympus AU480 biochemical analyzer;

c) immunologic indicators included determining immunoglobulins on the Minicap and Sebia analyzer, determining bactericidal activity of blood serum using Staphylococcus epidermidis test culture modified according to Smirnov and Kuzmina (1966) as well as lysozyme activity, using a Micrococcus lysodeikticus test culture in the modification of USRIEVM (Ukrainian Scientific-Research Institute of Experimental Veterinary Medicine).

The analyses were performed in the "Hemohelp" laboratory of the Department on "Anatomy, Surgery and Internal Noncommunicable Diseases" at Nizhny Novgorod State Agricultural Academy, and in the laboratory of protein-amino acid nutrition of All-Russia Institute of Animal Physiology, Biochemistry and Nutrition.

The resulting digital material was subjected to statistical processing using conventional parametric methods. The degree of confidence was determined by Student's t-test employing the Microsoft Excel (2000) software package and STAT 3 software program.

\section{RESULTSAND DISCUSSION}

Analyzing the data obtained in the $1^{\text {st }}$ stage of the experiment, it is noted that a day after the start of feeding calves with colostrum, the level of immunoglobulins in the blood of newborn calves has increased by $32.4 \%$, total protein - by $5.6 \%$, and hemoglobin - by $3.9 \%$ as compared with the calves in the control group, where nursing cows were not administered timogen. These differences were preserved to a certain extent 10 days after calves birth as well. Hematological parameters were statistically processed, summarized and presented in Table 1.

Average daily gain of live weight of calves from the experimental group after two-month rearing amounted to $472 \mathrm{~g} /$ day versus $413 \mathrm{~g}$ /day in the control calves; that is, the increase amounted to $14.3 \%$.

Analyzing the data obtained in the $2^{\text {nd }}$ stage of the experiment, it was noted that one day after birth and feeding calves with colostrum, the calves born from cows in the experimental group, 
have shown insignificant increase of the number of erythrocytes (by 16.1\%) and leucocytes (by $15.9 \%$ ) in blood, as well as decrease in the number of monocytes in comparison with control animals. At that, the number of leukocytes was higher mainly due to lymphocytes, and to a lesser extent due to the number of neutrophils. Hematological parameters of calves' blood in the $2^{\text {nd }}$ stage of the experiment were statistically processed, summarized and presented in Table 2.

Table 1. Hematological parameters of calves’ blood

\begin{tabular}{lccccc}
\hline \multirow{2}{*}{ Indicator } & \multicolumn{2}{c}{ Twenty-four-hours old calves } & & \multicolumn{2}{c}{ Ten-days-old calves } \\
\cline { 2 - 3 } \cline { 5 - 5 } & Control & Experiment & & Control & Experiment \\
\cline { 5 - 6 } Immunoglobulins, $\mathrm{mg} / \mathrm{ml}$ & $10.8 \pm 1.2$ & $14.3 \pm 1.1^{*}$ & & $11.7 \pm 0.9$ & $13.8 \pm 1.3$ \\
Total protein, g/l & $57.1 \pm 4.3$ & $60.3 \pm 5.6$ & & $59.2 \pm 6.1$ & $61.5 \pm 4.7$ \\
Hemoglobin, g/l & $89.2 \pm 7.6$ & $92.7 \pm 9.4$ & & $92.3 \pm 7.9$ & $94.2 \pm 8.5$ \\
Blood urea, mmol/l & $4.9 \pm 0.5$ & $5.2 \pm 0.4$ & & $4.8 \pm 0.4$ & $4.5 \pm 0.3$ \\
Glucose, $\mathrm{mmol} / \mathrm{l}$ & $5.6 \pm 0.5$ & $5.9 \pm 0.5$ & & $5.3 \pm 0.5$ & $5.2 \pm 0.5$ \\
\hline
\end{tabular}

$* \leq 0.05$ as compared to control

Table 2. Hematological parameters of calves' blood ( $2^{\text {nd }}$ stage of the experiment)

\begin{tabular}{|c|c|c|c|c|}
\hline \multirow[t]{2}{*}{ Indicator } & \multicolumn{2}{|c|}{ Twenty-four-hours old calves } & \multicolumn{2}{|c|}{ Ten-days-old calves } \\
\hline & Control & Experiment & Control & Experiment \\
\hline Erythrocytes, million/ $\mu l$ & $5.0 \pm 0.4$ & $5.8 \pm 0.4$ & $4.9 \pm 0.3$ & $5.49 \pm 0.5$ \\
\hline Leukocytes, thousand/ $\mu \mathrm{l}$ & $4.6 \pm 0.2$ & $5.3 \pm 0.3$ & $4.7 \pm 0.4$ & $5.22 \pm 0.4$ \\
\hline Banded neutrophil, \% & $2.1 \pm 0.1$ & $3.0 \pm 0.4$ & $2.7 \pm 0.3$ & $2.9 \pm 0.2$ \\
\hline Segmentonuclear neutrophils, \% & $27.3 \pm 1.9$ & $24.8 \pm 3.2$ & $26.1 \pm 2.5$ & $25.3 \pm 2.8$ \\
\hline Eosinophils, \% & 0.8 & 1.2 & 1.0 & 1.8 \\
\hline Monocytes, \% & 4.0 & 0.8 & 3.6 & 2.8 \\
\hline Lymphocytes, \% & $68.8 \pm 4.9$ & $70.2 \pm 5.5$ & $66.5 \pm 4.3$ & $67.2 \pm 7.2$ \\
\hline The total number of neutrophils, thousand/ $\mu \mathrm{l}$ & 1.6 & 1.5 & 1.4 & 1.4 \\
\hline The total number of lymphocytes, thousand/ $\mu \mathrm{l}$ & 3.0 & 3.7 & 3.1 & 3.5 \\
\hline The ratio of lymphocytes/segmentonuclear neutrophils & 2.4 & 2.8 & 2.6 & 2.7 \\
\hline The ratio of neutrophils/lymphocytes & 0.5 & 0.4 & 0.3 & 0.4 \\
\hline
\end{tabular}

* $\leq 0.05$ as compared to control

Table 3. Immunological and biochemical parameters of calves' blood ( $2^{\text {nd }}$ stage of the experiment)

\begin{tabular}{|c|c|c|c|c|}
\hline \multirow[t]{2}{*}{ Indicator } & \multicolumn{2}{|c|}{ Twenty-four-hours old calves } & \multicolumn{2}{|c|}{ Ten-days-old calves } \\
\hline & Control & Experiment & Control & Experiment \\
\hline Hemoglobin, g/l & $95.8 \pm 8.5$ & $99.7 \pm 7.6$ & $94.7 \pm 8.1$ & $97.9 \pm 9.1$ \\
\hline Blood urea, mmol/l & $5.4 \pm 0.6$ & $5.6 \pm 0.5$ & $5.2 \pm 0.5$ & $5.3 \pm 0.4$ \\
\hline Glucose, mmol/l & $6.1 \pm 0.4$ & $5.9 \pm 0.4$ & $5.9 \pm 0.4$ & $5.5 \pm 0.6$ \\
\hline Total protein, g/l & $57.6 \pm 4.6$ & $60.8 \pm 3.7$ & $58.9 \pm 5.3$ & $61.7 \pm 4.1$ \\
\hline Protein fractions: albumins, g/l & $18.5 \pm 1.1$ & $17.8 \pm 1.5$ & $20.0 \pm 1.6$ & $19.3 \pm 1.3$ \\
\hline$\alpha$-globulins, g/l & $16.4 \pm 1.3$ & $15.6 \pm 1.2$ & $15.1 \pm 0.9$ & $14.2 \pm 1.5$ \\
\hline$\beta$-globulins, g/l & $9.3 \pm 0.5$ & $10.8 \pm 0.9$ & $9.1 \pm 1.3$ & $10.3 \pm 1.0$ \\
\hline$\gamma$-globulins, g/l & $13.4 \pm 0.8$ & $16.6 \pm 0.7 *$ & $14.8 \pm 1.5$ & $17.7 \pm 1.8$ \\
\hline Bactericidal activity, \% & $36.3 \pm 2.8$ & $39.8 \pm 4.2$ & $38.5 \pm 3.4$ & $40.7 \pm 2.9$ \\
\hline Lysozyme activity, \% & $17.3 \pm 1.9$ & $18.8 \pm 1.5$ & $18.6 \pm 2.0$ & $19.4 \pm 1.6$ \\
\hline
\end{tabular}

$* \leq 0.05$ as compared to control 
Apparently, a change in the content of neutrophils and lymphocytes has led to a change of nonspecific reactivity indicators. Thus, the index of lymphocytes/segmentonuclear neutrophils has increased by $29 \%$, while index of neutrophils/ lymphocytes has decreased by $11.1 \%$.

After twenty-four hours from starting feeding with colostrum, the calves born from cows, which were subjected to intramuscular injection of timogen at a dose of $1.2 \mathrm{mg}$ per animal once a day 3-6 days before calving, have shown a higher level of total protein (by 5.5\%) and its fractions â- and ã-globulins in blood, which exceeded control by 9.9 and $17.7 \%$, respectively. Bactericidal activity of blood serum, reflecting the cumulative effect of humoral and cellular protection components, was significantly above controls in calves of the experimental group (by 9.6\%), at that lysozyme activity increased by $8.7 \%$.

The revealed differences of morphological indicators of blood in calves involved in the $2^{\text {nd }}$ stage of the experiment were reflected in biochemical and immunological parameters of blood of these animals and largely preserved during 10 days after birth. The observed change in blood indicators of calves in the experimental group is probably associated with revealed high content of immunoglobulins in colostrum of cows, which were administered timogen before calving. Thus, in experimental calves their level amounted to $52.1 \mathrm{mg} / \mathrm{ml}$ versus $43.2 \mathrm{mg} / \mathrm{ml}$ in control, while lymphocytes exceeded control by $7.2 \%$. However, intake with colostrum of larger quantities of other factors enhancing pinocytosis in the gut of the calves from experimental group cannot be excluded. Immunological and biochemical parameters of blood are statistically processed, summarized and presented in Table. 3.

Stimulation of immunoglobulins and leucocytes separation out of colostrum as well as changes in other immunogenic factors, have contributed to the increase in the level of metabolic and immunological status of calves. This has led to the decrease of their morbidity and increase in weight gain by $17.6 \%$ as compared to the control, i.e. by 537 and $458 \mathrm{~g} /$ day in the experimental and control groups, respectively, in the dairy cultivation period during two-month observation.

\section{CONCLUSION}

Correction of metabolic and immune processes in the body is a relevant, complex and extensive problem in humane medicine, which requires scientifically-based practical solution.

A single intramuscular injection of the timogen aqueous solution to down-calving cows 3-6 days before calving contributed to the accumulation in the mammary gland of immunoglobulins and other immunogenic factors. The amount of immunoglobulins in the colostrum of the tested cows, equal to 52.1 versus $43.2 \mathrm{mg} / \mathrm{ml}$ and a number of lymphocytes greater at $7.2 \%$, helped to improve immunological status and metabolic processes in the organism of newborn calves after feeding them with colostrum that was clearly reflected by hematological status. A numbers of red blood cells and white blood cells were increased mainly due to lymphocytes, as well as a higher level of protein and its fractions, especially â- and ã- globulins were observed compared to the control. Also tested calves had higher bactericidal and lysozyme activity of blood serum.

It is found in general that the injection of timogen to down-calving cows contributes to the accumulation of immunoglobulins and other immunogenic factors in the mammary gland of cows before calving and their release with colostrum that promotes the formation of highlevel metabolic and immunological status of newborn calves after feeding them with colostrum.

The data obtained allow clarifying some aspects of immune status and metabolism regulation in the organism of newborn calves that must be taken into account when developing physiologically sound practical ways of immunomodulation and improvement of metabolic processes in calves organism during the initial period of nurturance, which is often accompanied by immunodeficiency and diseases of the gastrointestinal tract of these animals.

\section{REFERENCES}

1. Vorobyev, A.A., Lyashchenko, V.A., "Immunobiological Preparations: Present and Future,” Journal of Microbiology, Epidemiology and Immunology, 1995; 6: 105-111. 
2. Kovalenko, Ya.R., "Formation of the Immunological Status of Young Farm Animals," Bulletin of Agricultural Science, 1979; 50-58.

3. Smirnov, V.S., "Timogen in Animal Husbandry and Veterinary Science,” St. Petersburg, Medical and Biological Scientific-Industrial Complex, 2005; 36.

4. Kharitonov, L.V., Moseeva, A.I., Velikanov, V.I., Kharitonova, O.V., and Kaurkina E.V., "The Influence of Dipeptide Timogen and its Combination with Leucopoiesis Stimulator on the Absorption of Immunoglobulins in Newborn Calves,” Veterinary Physician, 2014; 3: 33-38.

5. Karput, I.M., and Pivovar, L.M., "Immunology of Lactation in Autoimmune Diseases and its Role in the Etiopathogenesis of Dyspepsia of Newborn Calves," Bulletin of the National Academy of Sciences of Belarus, Agricultural Sciences, 1982; 4: 108-110.

6. Morozov, V.G., and Khavinson, V.K., "Natural and Synthetic Thymus Peptides as Therapeutics for Immune Dysfunction,” Int. Immunopharm, 1997; 19: 501-505.

7. Zenkin, A.S, Kalyazina, N.Y., Eryashev, P.N., Pilgaev, F.P., and Switin A.I., "Scientific and Experimental Substantiation of Possibility of Application of the Original Drug Bio-effective W in Bovine Leucosis," Research Journal of Pharmaceutical, Biological and Chemical Sciences , 2016; 7(2), pp. 1040-1045.

8. Khavinson, V.Kh., and Anisimov, V.N., "Peptide
Bioregulators and Aging," St. Petersburg, Science, 2003; 223.

9. Velikanov, V.I., Shumov, I.S., Maslova, M.A., and Kharitonov, L.V., "The State of Nonspecific Resistance of Newborn Calves under the Influence of Amino Acid Preparations," Proceedings of the $18^{\text {th }}$ Int. Conf. "New Pharmacological Agents in Veterinary Medicine,” St. Petersburg, 2006; 49-50.

10. Belokrylov, G.A., Molchanova, I.M., and Sorochinskaya, E.I., "Amino Acids as Immunogenesis Stimulators,” Bulletin of the USSR Academy of Sciences, 1986; 2: 289-293.

11. Khavinson, V.Kh., Kvetnoy, I.M., and Ashmarin, I.P., "Peptidergic Regulation of Homeostasis," Successes of Modern Biology, 2002; 41: 83-96.

12. Asatiani, V.S., "New Methods of Biochemical Photometry,” Moscow, 1965; 543.

13. Kondrakhin, I.P., Kurilov, N.V., Malakhov, A.G., et al., "Clinical Laboratory Diagnostics in Veterinary Medicine,” Reference Book, Moscow, Agropromizdat 1985; 287 p.

14. Goldstein, A.L., Cohen, G.H., and Zatz, M.M., 1972, "Purification and Biological Activity of Thymosin, a Harmone of Thymus Gland," Proceedings of Natl. Acad. Sci. USA, 69, pp. 1800-1803.

15. Zenkin, A.S, Kalyazina, N.Y., and Switin, A.I., 2014, "Experimental Evaluation of the Feasibility of the Cerebrospinal Fluid for Stimulation of the Erytropoietic Hematopoietic Lineage,” Biosciences Biotechnology Research Asia, 11, pp. 215-219. 